\title{
Computer Analysis of Electromagnetic Transients in Grounding Systems Considering Variation of Soil Parameters with Frequency
}

\author{
Marco A. O. Schroeder ${ }^{1 *}$, Márcio M. Afonso², Tarcísio A. S. Oliveira ${ }^{2}$, Sandro C. Assis ${ }^{3}$ \\ ${ }^{1}$ Department of Electrical Engineering, Federal University of São João del-Rei, Laboratory of Applied Electromagnetism, São João \\ del-Rei, Brazil; ${ }^{2}$ Department of Electrical Engineering, Federal Center of Technological Education of Minas Gerais, Belo Horizonte, \\ Brazil; ${ }^{3}$ Energetic Company of Minas Gerais, Belo Horizonte, Brazil.

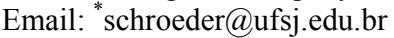

Received September $15^{\text {th }}, 2012$; revised October $14^{\text {th }}, 2012$; accepted October $25^{\text {th }}, 2012$

\begin{abstract}
This paper presents a mathematical model to calculate transients in grounding systems. The derived equations arise from direct application of basic electromagnetic equations in frequency domain, whose solution is obtained by the application of the Moment Methods. A formulation based on experimental measurements is applied to quantify the soil parameters for each frequency. The unified approach is applied in the calculation of the grounding impedance of horizontal electrodes. Results show that the inclusion of frequency dependence of the soil parameters leads to a reduction of the values of grounding impedance, in comparison with results for soils with parameters independent of frequency.
\end{abstract}

Keywords: Grounding Electrodes; Grounding Impedance; Transient Response; Frequency Response; Electromagnetic Modeling

\section{Introduction}

The grounding systems are an important element for good electrical systems performance, mainly when they are subjected to faults. Their basic function is to disperse the current of fault to earth without causing any potential differences or induced voltages that might endanger people or damage equipments. Grounding performance and design at low frequencies are well established and described in international standards [1]. However, when energized by lightning currents, they present a very particular behavior and their analysis cannot be carried out with traditional methodologies employed in low frequency occurrences [2].

The transient analysis of grounding electrodes is usually developed based on three main different approaches: 1) electromagnetic field theory [3]; 2) transmission line theory [4], and 3) circuit theory [4]. The first one is considered as the most accurate since it is based on least neglects in comparison to the methods based on transmission line and circuit theory.

Further, in the grounding study, it is of major importance the adequate soil modeling [5-13]. In most works dealing with lightning transients in grounding, the soil electrical conductivity and permittivity are usually assumed to be frequency independent (e.g. in $[3,4,14]$ ).

"Corresponding author.
Typically the soil conductivity is assumed as the value measured by conventional measuring instruments, which employ low frequency signals [2]. In the same approach, the soil relative permittivity is assumed to vary from 4 to 81 , depending on the soil humidity [2]. However, measurements of the soil electromagnetic behavior show that both parameters are strongly frequency dependent [6-13]. Experimental data obtained in [6-12] for a large number of soil samples indicate an increase of the soil conductivity and a reduction of the relative permittivity when frequency rises from about $100 \mathrm{~Hz}$ to $2 \mathrm{MHz}$. An explanation physically consistent of these behaviors is described in [13]. The soil magnetic permeability is, in general, equal to vacuum magnetic permeability [6].

In this work a methodology based on the electromagnetic field theory and Moment Method is proposed to evaluate the impulse behavior of grounding. The transient is solved in the frequency domain. Proper relations to quantify the soil electric conductivity and permittivity for each frequency are employed. The developed methodology is applied in the calculation of the grounding impedance of horizontal electrodes.

This paper presents two main contributions in the analyses of grounding transients. First, the presented results are based on an accurate mathematical model, which take into account the propagation effects and electromagnetic 
interaction between the grounding elements. Second, a formulation to quantify the frequency dependence of the soil parameters is included and an evaluation of the influence of such dependence is developed.

This article is organized as follows: presented in Section 2 the electromagnetic grounding model; in Section 3 is described the soil modeling; the results are presented, discussed and interpreted in Section 4 and finally in Section 5 outlines the conclusions.

\section{The Electromagnetic Grounding Model}

The Hybrid Electromagnetic Model (HEM) was used to simulate the grounding behavior. The formulation of this model was first proposed by Visacro and Portela [15], who developed a frequency-domain model to address the transient response of grounding electrodes using Fourier Transform. This model employs the scalar electric potential and the vector magnetic potential to take into account the electromagnetic coupling between the grounding elements. Later, this formulation evolved to the so-called HEM model, detailed by Visacro and Soares in [16], to address the simulation of general lightning related engineering problems, such as overvoltages developed by direct lightning strikes and voltages induced by nearby strikes $[17,18]$.

The electromagnetic model is based on the fundamental idea of represent a current-carrying conductor as a source of transversal and longitudinal currents. The transversal current crosses the conductor surface and is spread into the surrounding medium and the longitudinal current flows along the conductor. Such idea was first explored in [16] to model cylindrical conductors carrying lightning currents. Here, it is applied in modeling grounding systems under transient conditions.

The grounding system is represented by a set of cylindrical electrodes immersed in the soil. Each electrode is source of a transversal current IT and a longitudinal current IL, as illustrated in Figure 1. The current IT generates a divergent electric field at a generic point that establishes a potential rise in relation to remote earth in such point [19]. Considering each pair of electrodes, as illustrated in Figure 2, this current yields capacitive and conductive coupling (self and mutual ones). The current IL generates a nonconservative electric field, which integration between two different points results in a voltage drop along the defined path [19]. Considering each pair of electrodes, as illustrated in Figure 2, this current yields inductive coupling (self and mutual ones).

The electromagnetic coupling between the electrodes can be computed by electromagnetic fields integration along each one and leads to the following equations [16]: where $V_{i j}$ refers to average potential of electrode $i$ due to the transversal current dispersed by electrode $j ; \Delta V_{i j}$ is

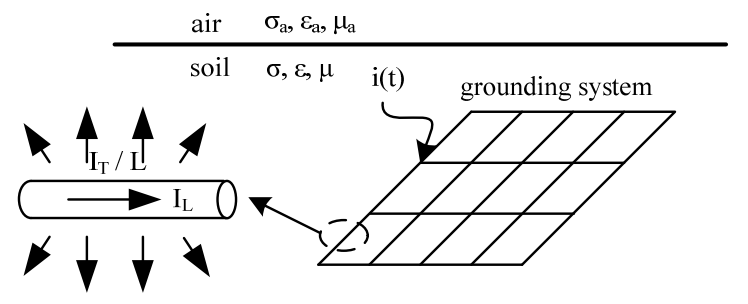

Figure 1. Grounding system representation.

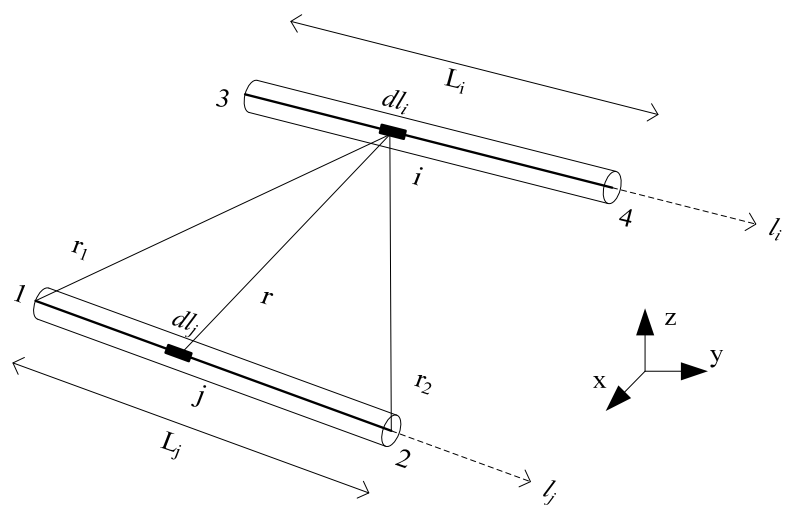

Figure 2. Electrodes interaction.

$$
\begin{gathered}
V_{i j}=\frac{1}{4 \pi(\sigma+j \omega \varepsilon) L_{j} L_{i}} \int_{L_{i}} \int_{L_{j}} I_{T j} \frac{\mathrm{e}^{-\gamma r}}{r} \mathrm{~d} l_{j} \mathrm{~d} l_{i}, \\
\Delta V_{i j}=-j \omega \frac{\mu}{4 \pi} \int_{L_{i} \int_{L_{j}}} I_{L j} \frac{\mathrm{e}^{-\gamma r}}{r} \mathrm{~d} l_{j} \cdot \mathrm{d} l_{i},
\end{gathered}
$$

the voltage drop along electrode $i$ due the longitudinal current flowing along electrode $j ; \sigma, \varepsilon$ and $\mu$ are, respectively, the medium electric conductivity, electric permittivity and magnetic permeability; $\gamma$ is the propagation constant and $\omega$ is the angular frequency.

The final solution is obtained by the application of Moments Method (MoM) [20]. The electrode (length $L$ ) is divided into $N$ elements of length $\ell=L / N$. The value of $N$ is determined in a way that the thin-wire approximation is valid for the longitudinal current along the element. Also, the length of each element is sufficiently small, so that the longitudinal current along it may be considered uniform. In this way, the longitudinal current is represented by a piecewise-constant distribution. In a similar approach the current that diverges from each element is assumed to be uniform along it. The choice of these basis functions to represent $I_{T}$ and $I_{L}$ leads to a straightforward physical interpretation. The longitudinal current of an element $N$ is exactly that of the element $N$-1 subtracted the transversal current dispersed to the soil from this last one.

From the above considerations, the transversal $I_{T j}(\ell)$ and the longitudinal $I_{L j}(\ell)$ current distributions along 
the electrode are represented by a linear combination of $N$ basis function $P_{n}(\ell)$, or:

$$
\begin{aligned}
& I_{T j}(\ell)=\sum_{n=1}^{N} I_{T n} P_{n}(\ell), \\
& I_{L j}(\ell)=\sum_{n=1}^{N} I_{L n} P_{n}(\ell),
\end{aligned}
$$

where,

$$
P_{n}(\ell)= \begin{cases}1 & \text { on the } n \text {-th element; and } \\ 0 & \text { otherwise }\end{cases}
$$

In Equations (3) and (4), $I_{T n}$ and $I_{L n}$ are unknown coefficients and correspond, respectively, to the transversal and longitudinal current of the $n$-th element. To determinate such coefficients $N$ independent linear equations are necessary. These equations are obtained by the substitution of Equation (3) in Equation (1) and Equation (4) in Equation (2) and by considering the self and mutual interactions between the $N$ elements. From that two systems of linear equations can be obtained: $V=Z_{T} I_{T}$ and $\Delta V=Z_{L} I_{L}$. The terms of $V$ and $I_{T}$ correspond, respectively, to the average potential and transversal current in each element and the terms of $Z_{T}$ are defined as the transversal impedance between the elements $i$ and $j$. The terms of $\Delta V$ and $I_{L}$ correspond, respectively, to the voltage drop and longitudinal current in each element and the terms of $Z_{L}$ are defined as the longitudinal impedance between the elements $i$ and $j$. In $Z_{T}$ and $Z_{L}$ computation, the influence of air-soil interface is taken into account by means of the modified image theory [21]. In this case, the image source is symmetrically positioned with reference to real source and has magnitude equal to the real one multiplied by a coefficient, which depends on the electromagnetic characteristics of air and soil [21]. The reciprocity theorem is valid and, thus, the $Z$-matrices are symmetrical and only half of the elements have to be evaluated, what permits a substantially reduction of computational time. The two systems of linear equations may be reduced to a unique system by means of two fundamental considerations [16,19]: 1) the average potential in each element is equal to the arithmetic media of nodal potentials and the voltage drop in each element is equal to the difference of nodal potentials; 2) the Kirchhoff's current law is explicitly enforced over all element's nodes. Considering these two assumptions, an only system of linear equations $A V_{N}$ $=b$ is composed [16,19]. The matrix $A$ is a composition of original $Z$-matrices, the vector $V_{N}$ corresponds to nodal potentials and $b$ refers to external current injected into each node (null value for nodes with no current injection). By the system solution, all nodal potentials are determined and transversal and longitudinal currents of each element can be immediately calculated. From these variables, other quantities may be obtained, as the potential in node of current injection and the grounding impedance.

The described procedure provides solution for only one frequency. The solution must be determined for a range of frequencies, which depends of the analyzed problem (short circuit or lightning discharge, for example). From the frequency response, the time domain response may be obtained by application of the inverse Fourier transform. The main motivation to solve the transient in frequency domain is to include the frequency dependence of the soil parameters [13].

\section{Soil Modeling}

The presented work considers a formulation for expressing the frequency dependence of the soil parameters $(\sigma$ and $\varepsilon$ ) originated from field measurements [6-9]. A very large number of different soil and geological structures in Brazil were tested in the frequency range of $100 \mathrm{~Hz}$ to 2 $\mathrm{MHz}$, as described in [6-9]. After the measurements, the soil samples were fitted to a curve assuming [6-9]:

$$
\sigma+j \omega \varepsilon=\sigma_{0}+\Delta i\left[\operatorname{cotang}\left(\frac{\pi}{2} \alpha\right)+j\right]\left(\frac{\omega}{2 \pi \times 10^{6}}\right)^{\alpha},
$$

where $\sigma_{0}$ is the electric conductivity at low frequency and $\Delta i$ and $\alpha$ are statistical parameters, which are responsible for the frequency dependence of soil conductivity and permittivity [6-9]. To evaluate the probability density functions associated with parameters $\Delta i$ and $\alpha$, Weibull approximations were considered. As discussed in [6-9], for most purposed, it may be acceptable to consider median values for both $\Delta i$ and $\alpha$, which are, respectively, $11.71 \mathrm{~S} / \mathrm{m}$ and 0.706 . Only as a reference, considering the median values, Equation (6) indicates a variation of $\varepsilon_{r}$ from about 3000 to 170 , when frequency varies from 100 $\mathrm{Hz}$ to $2 \mathrm{MHz}$. In the same frequency range, a soil with $\sigma_{0}$ $=1 \mathrm{mS} / \mathrm{m}$ has it conductivity increased to about $6 \sigma_{0}$. It is important to observe that even though $\varepsilon$ decreases with frequency, the relation $\omega \varepsilon$ still increases with the frequency rise. Further details can be seen in [13].

A valuable aspect of the presented expression is that it was developed from measurements performed in field conditions and with the natural soil humidity in contrast with the laboratorial experiments of the classical works $[10,11]$. A detailed description of the measurement procedure is presented in [6-9], including experimental setup, methods for soil sampling, and checking physical consistency.

It is worth to mentioning that such formulation has been extensively used in the last few years to investigate the influence of frequency dependent soil parameters in transmission line modeling, for example, in [7-9]. Also, has been used to investigate the influence of frequency dependent soil parameters in electric fields of grounding 
electrodes in [13]. Nevertheless, the impact of the frequency dependence of soil parameters in the grounding impulse behavior is still an open issue. The next section presents a preliminary analysis of such impact, considering the calculation of the grounding impedance of some simple electrode configurations. In simulations, the variation of soil parameters with frequency is computed according to Equation (6).

\section{Results}

\subsection{System under Study}

The evaluated grounding configuration corresponds to horizontal electrodes, buried in a depth of $0.5 \mathrm{~m}$, with radius of $1 \mathrm{~cm}$ and of lengths ranging from 5 to $80 \mathrm{~m}$.

Two soil models were adopted, that is: Soil $1-$ Soil represented by its low-frequency electric conductivity $\sigma_{0}$ (three representative values considered in this work: 10, 2 and $1 \mathrm{mS} / \mathrm{m}$ ) and relative permittivity equal to 15 , both parameters frequency independent. Soil 2-Soil with the inclusion of frequency-dependent parameters and the same previous low-frequency conductivity values. In simulations, the effects of the soil ionization are disregarded.

The electrode excitation was obtained by the injection of a typical double exponential current wave of $1 \mathrm{kA}$ and $1.2 / 50 \mu \mathrm{s}$. In all simulations, the current injection was made in the electrode termination.

The following definitions for the analyzed quantities are adopted in this paper:

- Harmonic impedance: $Z(j \omega)=V(j \omega) / I(j \omega)$, where $I(j \omega)$ and $V(j \omega)$ are phasors of the injected current and of the potential at the injection point, respectively;

- Impulse impedance: $Z_{p}=V_{p} / I_{p}$, where $V_{p}$ is the peak value of the transient voltage at the injection point and $I_{p}$ is the peak value of the injected current.

\subsection{Harmonic Impedance}

To evaluate the influence of the soil parameters variation with frequency on the harmonic grounding impedance, a $60-\mathrm{m}$ long horizontal electrode is considered, buried in both Soil 1 and 2, with $\sigma_{0}=10,2$ and $1 \mathrm{mS} / \mathrm{m}$. Figure 3 illustrates the amplitude $[|Z(\omega)|$, Figure 3(a)], and the angle $[\theta(\omega)$, Figure $3(\mathbf{b})]$, of the harmonic grounding impedance $Z(\omega)$. The harmonic impedance is frequency independent and equal to the low frequency ground resistance R, in the Low Frequency (LF) range, for both Soil 1 and 2. Nevertheless, as frequency rises, a different behavior of $Z(\omega)$ is observed depending on the soil model. Results for Soil 1 exhibit an inductive behavior and the amplitude of $Z(\omega)$ becomes larger than $\mathrm{R}$, being this ef-

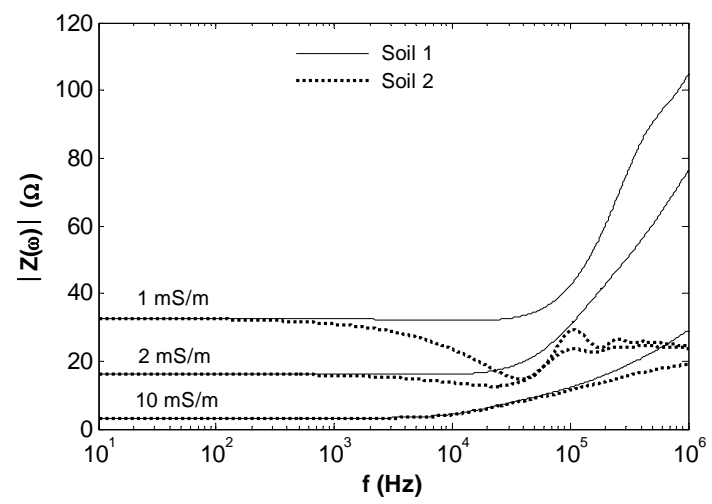

(a)

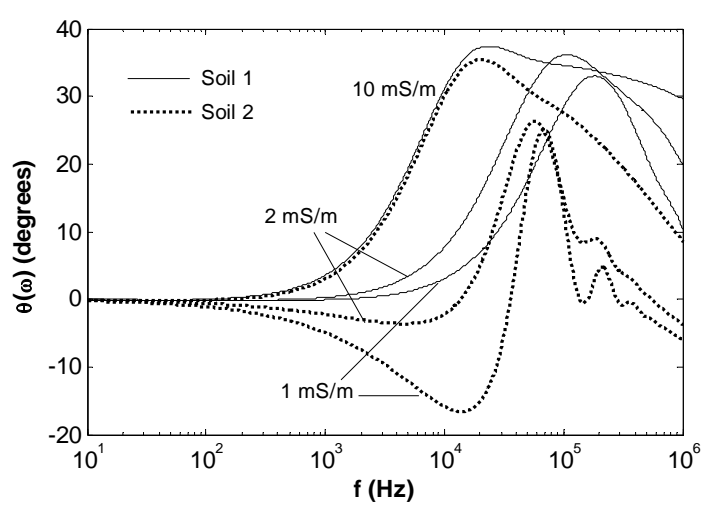

(b)

Figure 3. 60 m horizontal electrode. (a) Amplitude; (b) Angle of the harmonic grounding impedance.

fect more relevant for less conductive soils. These results are in perfect consonance with some classical ones, for example, those presented by Grcev in [14]. On the other hand, when the soil parameters dependence with frequency is considered (Soil 2), the capacitive effect becomes relevant, especially for high-resistivity soils $[\theta(\omega)$ $<0$, Figure 3(b)]. The capacitive effect plays an important role in the grounding performance and is responsible for the reduction of $|Z(\omega)|$. Indeed, as may be observed in Figure 3(a), the frequency dependence of the soil parameters leads to reduction of the amplitude values of $Z(\omega)$ in the High Frequency (HF) range.

\subsection{Impulse Impedance}

Figure 4 shows the simulation results for the low frequency ground resistance $\mathrm{R}$ and the impulse impedance $Z_{p}$ of horizontal ground electrodes in a range from 5 to 80 $\mathrm{m}$, for both Soil 1 and 2, with $\sigma_{0}=10,2$ and $1 \mathrm{mS} / \mathrm{m}$. According to Figure 4, the value of the ground resistance is larger for less conductive soils and decreases with increasing the electrode length. Similarly, the impulse impedance, for both soil models, presents larger values for less conductive soils and decrease with increasing the 


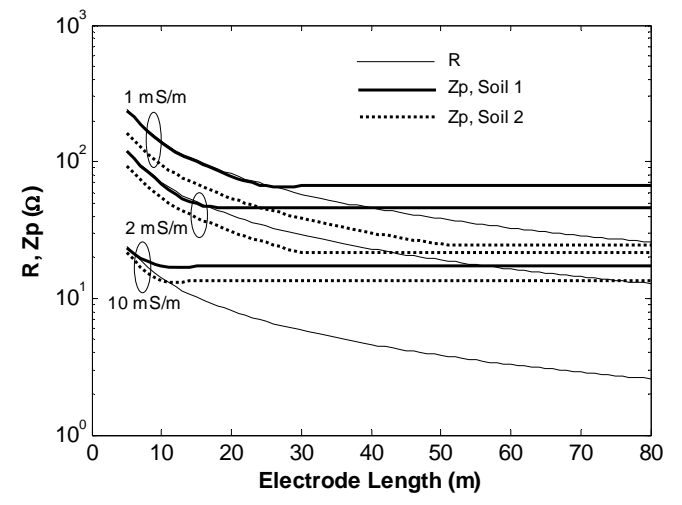

Figure 4. LF ground resistance $R$ and impulse impedance $Z_{p}$ of horizontal ground electrode of $5-80 \mathrm{~m}$, in both Soil 1 and 2 , and $\sigma_{0}=10,2$ and $1 \mathrm{mS} / \mathrm{m}$.

electrode length. Nevertheless at a certain length it becomes constant, while the LF resistance continues to decrease. Therefore, only a certain electrode length is effective in controlling the impulse impedance, which is referred as effective length $\ell_{e f}$ [14]. The effective length decreases with soil conductivity $\sigma_{0}$ and frequency rise. This can be understood as both parameters are responsible for increasing ground losses, leading to an increase in the attenuation of the current wave that propagates along the electrode [2]. It means that as the wave is attenuated, the electrode length that is effectively used to disperse the lightning current is reduced.

Figure 4 also shows two very important differences between simulated results for Soil 1 and 2. First, it may be observed that the effective length is larger for a soil with frequency-dependent parameters (Soil 2). This effect is more appreciable in less conductive soils and probably is related to the reduction of the attenuation constant of soil in the intermediate frequency range, when its parameters are assumed to be frequency dependent. Second, the values of impulse impedance for soil with frequency variation of its parameters (Soil 2) are lower than that obtained for soil represented by only its LF parameters (Soil 1), especially for high-resistivity soils. Such difference between the obtained values becomes larger above the effective length for Soil 1, since the impulse impedance continues to decrease for Soil 2. As a conclusion, a significant reduction of the overvoltages, due to the soil parameters variation with frequency, is expected for electrodes lengths larger than the effective one for Soil 1.

\section{Conclusions}

An accurate methodology to calculate transients in grounding systems, which include the frequency dependence of the soil parameters, is proposed. Results show that:

1) The inclusion of frequency dependence of the soil parameters leads to a reduction of the values of grounding impedance and a increasing of the values of effective length, in comparison with results for soils with parameters independent of frequency, especially for high-resistivity soils;

2) The consideration of frequency independent soil parameters leads to conservative values of grounding impedance. So, new investigations are still necessary considering other formulations to determinate soil parameters in function of frequency.

\section{Acknowledgements}

The authors gratefully acknowledge the financial support provided by Energetic Company of Minas Gerais (CE$\mathrm{MIG)}$ and also the electrical engineer R. S. Alípio for valuable discussions and contributions.

\section{REFERENCES}

[1] The IEEE Standards Association, "IEEE Guide for Safety in AC Substation Grounding," The IEEE Standards Association, New York, 2000.

[2] S. Visacro, "A Comprehensive Approach to the Grounding Response to Lightning Currents," IEEE Transactions on Power Delivery, Vol. 22, No. 1, 2007, pp. 381-386. doi:10.1109/TPWRD.2006.876707

[3] L. Grcev and F. Dawalibi, "An Electromagnetic Model for Transients in Grounding Systems," IEEE Transactions on Power Delivery, Vol. 5, No. 4, 1990, pp. 1773-1781. doi: $10.1109 / 61.103673$

[4] Y. Liu, N. Theethayi and R. Thottappillil, "An Engineering Model for Transient Analysis of Grounding System Under Lightning Strikes: Nonuniform Transmission-Line Approach," IEEE Transactions on Power Delivery, Vol. 20, No. 2, 2005, pp. 722-730. doi:10.1109/TPWRD.2004.843437

[5] A. F. Otero, J. Cidrás and J. L. del Álamo, "FrequencyDependent Grounding System Calculation by Means of a Conventional Nodal Analysis Technique," IEEE Transactions on Power Delivery, Vol. 14, No. 3, 1999, pp. 873878. doi:10.1109/61.772327

[6] C. M. Portela, "Measurement and Modeling of Soil Electromagnetic Behavior," Proceedings of IEEE International Symposium on Electromagnetic Compatibility, Seattle, 2-6 August 1999, pp. 1004-1009.

[7] C. M. Portela, M. C Tavares and J. Pissolato, "Accurate Representation of Soil Behaviour for Transient Studies," IEEE Proceedings Generation, Transmission and Distribution, Vol. 150, No. 6, 2003, pp. 736-744.

[8] C. M. Portela, J. B. Gertrudes, M. C. Tavares and J. Pissolato, "Earth Conductivity and Permittivity Data Measurements: Influence in Transmission Line Transient Performance," Elsevier, Electric Power Systems Research, Vol. 76, No. 11 2006, pp. 907-915. doi:10.1016/j.epsr.2005.11.006 
[9] A. C. S. Lima and C. M. Portela, "Inclusion of FrequencyDependent Soil Parameters in Transmission-Line Modeling," IEEE Transactions on Power Delivery, Vol. 22, No. 1, 2007, pp. 492-499. doi:10.1109/TPWRD.2006.881582

[10] C. L. Longmire and K. S. Smith, "A Universal Impedance for Soils,” Defense Nuclear Agency, Washington DC, 1975.

[11] J. H. Scott, "Electrical and Magnetic Proprieties of Rock and Soil," United States Department of the Interior Geological Survey, 1983.

[12] S. Visacro and R. S. Alípio, "Frequency Dependence of Soil Parameters: Experimental Results, Predicting Formula and Influence on the Lightning Response of Grounding Electrodes," IEEE Transactions on Power Delivery, Vol. 27, No. 2, 2012, pp. 927-935. doi:10.1109/TPWRD.2011.2179070

[13] R. S. Alípio, M. A. O. Schroeder, M. M. Afonso, T. A. S. Oliveira and S. C. Assis, "Electric Fields of Grounding Electrodes with Frequency Dependent Soil Parameters," Electric Power Systems Research, Vol. 83, No. 1, 2012, pp. 220-226. doi:10.1016/j.epsr.2011.11.011

[14] L. Grcev, "Impulse Efficiency of Ground Electrodes," IEEE Transactions on Power Delivery, Vol. 24, No. 1, 2009, pp. 441-451. doi:10.1109/TPWRD.2008.923396

[15] S. Visacro and C. M. Portela, "Soil Permittivity and Conductivity Behavior on Frequency Range of Transient Phenomena in Electric Power Systems," Proceedings of Symposium High Voltage Engineering, Braunschweig, 24-28
August 1987, pp. 107-112.

[16] S. Visacro and A. Soares, "HEM: A Model for Simulation of Lightning-Related Engineering Problems," IEEE Transactions on Power Delivery, Vol. 20, No. 2, 2005, pp. 1206 1208. doi:10.1109/TPWRD.2004.839743

[17] A. Soares, M. A. O. Schroeder and S. Visacro, "Transient Voltages in Transmission Lines Caused by Direct Lightning Strikes," IEEE Transactions on Power Delivery, Vol. 20, No. 2, 2005, pp. 1447-1452. doi:10.1109/TPWRD.2004.839214

[18] F. H. Silveira and S. Visacro, "The Influence of Attachment Height on Lightning-Induced Voltages," IEEE Transactions on Electromagnetic Compatibility, Vol. 50, No. 3, 2008, pp. 743-747. doi:10.1109/TEMC.2008.926885

[19] R. S. Alípio, M. A. O. Schroeder, M. M. Afonso and T. A. S. Oliveira, "Electromagnetic Fields of Buried Conductors," Proceedings of the International Conference on Grounding Earthing, Florianópolis, 11-15 November 2008, pp. 399-402.

[20] R. F. Harrington, "Field Computation by Moment Methods," IEEE Press, New York, 1993. doi: $10.1109 / 9780470544631$

[21] T. Takashima, T. Nakae and R. Ishibashi, "Calculation of Complex Fields in Conducting Media," IEEE Transactions on Electrical Insulation, Vol. EI-15, No. 1, 1980, pp. 1-7. doi:10.1109/TEI.1980.298290 